\title{
Escala de Metas de Realização no Trabalho: adaptação e evidências de validade
}

\author{
Lígia Carolina Oliveira-Silva ${ }^{1}$ \\ Universidade Federal de Uberlândia, Uberlândia-MG, Brasil \\ Juliana Barreiros Porto \\ Universidade de Brasília, Brasília-DF, Brasil
}

\section{RESUMO}

O objetivo do presente estudo consistiu em traduzir, adaptar e buscar evidências de validade para a Escala de Metas de Realização no Trabalho modelo $2 \times 2$. Participaram do estudo 358 trabalhadores, que responderam o instrumento com 23 itens. Os resultados da análise fatorial confirmatória indicaram que a escala com 18 itens apresentou melhor ajuste do modelo de quatro fatores (aproximação/ busca de maestria, evitação/fuga de maestria, aproximação/busca de desempenho, evitação/fuga de desempenho), o que vai ao encontro de estudos anteriores. Os índices de ajuste do modelo foram: $\mathrm{CMIN} / \mathrm{DF}=2,12 ; \mathrm{SRMR}=0,06 ; \mathrm{GFI}=0,92 ; \mathrm{CFI}=0,90 ; \mathrm{RMSEA}=0,05$. $\mathrm{O}$ instrumento apresenta índices adequados para uso em pesquisas sobre o tema e permite analisar as diferentes formas pelas quais um trabalhador busca o alcance de uma meta e qual orientação perpassa suas ações no trabalho.

Palavras-chave: metas; maestria; desempenho; trabalho.

\section{ABSTRACT - Goal Achievement at Work Scale: Adaptation and validity evidence}

This study aimed to translate, adapt and identify validity evidence for the $2 \times 2$ Framework of Goal Achievement at Work Scale. Participants comprised of 358 workers who responded to the 23-item instrument. Results from confirmatory factor analyses indicated that the 18-item scale presented better model fit than the four-factor model (mastery approach, mastery avoidance, performance approach, performance avoidance), which is consistent with previous studies. Model fit indices were: $\mathrm{CMIN} / \mathrm{DF}=2.12 ; \mathrm{SRMR}=0.06$; $\mathrm{GFI}=0.92 ; \mathrm{CFI}=0.90 ; \mathrm{RMSEA}=0.05$. The instrument presents appropriate indices for use in research on the topic and permits analysis of the different forms in which workers seek to achieve goals and which goal orientation permeates their actions at work. Keywords: goals; mastery; performance; work.

\section{RESUMEN - Escala de Metas de Logro en el Trabajo: adaptación y evidencias de validez}

El objetivo de este estudio fue traducir, adaptar y buscar evidencias de validez en la escala de Metas de Realización en el Trabajo Modelo 2x2. Los participantes del estudio fueron 358 trabajadores que respondieron el instrumento con 23 ítems. Los resultados del análisis factorial confirmatorio indicaron que la escala con 18 ítems presentó mejor ajuste del modelo de cuatro factores (aproximación/ búsqueda de maestría, evitación/fuga de maestría, aproximación/búsqueda de desempeño, evitación/fuga de desempeño), que corroboran estudios anteriores. Los índices de ajuste del modelo fueron: CMIN/DF=2,12; SRMR=0,06; GFI=0,92; CFI=0,90; RMSEA $=0,05$. El instrumento cuenta con índices adecuados para su uso en la investigación sobre el tema y permite analizar las diferentes formas en que un trabajador busca el alcance de una meta, y la orientación que posterga sus acciones en el trabajo. Palabras clave: metas; maestría; desempeño; trabajo.

A teoria da Orientação para Metas de Realização (Achievement Goal Orientation) representa uma das áreas mais ativas de pesquisa no campo de motivação e metas. Inicialmente, o conceito de orientação para metas teve como foco o contexto educacional/escolar, porém se expandiu rapidamente para outras áreas da Psicologia. No presente artigo, o foco será na Psicologia Organizacional e do Trabalho (POT), uma vez que o instrumento apresentado foi criado para ser utilizado no contexto laboral.
A proposição de tal instrumento é importante devido ao crescente número de publicações na literatura internacional e nacional sobre a teoria da Orientação para Metas de Realização (OMR). No Brasil, já existem estudos publicados sobre o tema, como o de Zenorini, Santos, e Bueno (2003), que apresenta o estudo de validação da Escala de Avaliação das Metas de Realização no contexto escolar; o de Valentini e Rudisill (2006), que faz uma discussão teórica acerca das pesquisas conduzidas em orientação para metas; e o de Santos, Alcará, e 
Monteiro (2012), que analisa a motivação para aprender à luz das teorias de metas de realização e teoria de autodeterminação. Ainda no contexto escolar, estudos de Santos com outros colaboradores (Santos, Alcará, \& Zenorini, 2013; Santos, Mognon, Alcará, \& Lemos, 2011; Zenorini \& Santos, 2010) têm aprofundado o tema da motivação para aprender em estudantes, principalmente por meio da proposição de instrumentos de medida e análise de suas propriedades psicométricas.

No contexto laboral, mediante consulta às bases de dados como Scielo, BVS-PSI e Google Acadêmico em janeiro de 2015, utilizando os termos "orientação para alcance de metas no trabalho", "metas de realização no trabalho", "orientação para metas de realização no trabalho" e "orientação para metas no trabalho", não foram encontrados trabalhos publicados sobre o tema, o que talvez possa ser explicado pela ausência de um instrumento de mensuração adequado ao contexto brasileiro. Diante dessa lacuna, o objetivo deste estudo consistiu em traduzir, adaptar e identificar evidências de validade para a escala de Baranik, Barron, e Finney (2007), intitulada Modelo 2x2 de Metas de Realização no Trabalho $(2 \times 2$ Framework of Achievement Goals for a Work Domain). Como será discutido mais à frente, essa escala representa o instrumento mais atual em relação ao avanço da teoria de alcance de metas.

\section{Orientação para metas de realização no trabalho: origens, definições e mensuração}

A teoria de Orientação para Metas de Realização (OMR) descreve como diferentes tipos de metas de realização podem influenciar certos resultados, tais como desempenho, especialmente no contexto educacional. A motivação para a realização de metas, especificamente as dimensões de busca/aproximação e fuga/evitação, representam a energização e direção do comportamento humano (Elliot, 2006). Esse movimento de aproximar-se ou evitar possibilidades positivas ou negativas assumiria duas formas distintas: (a) a promoção de novas situações positivas e manutenção das que já existam; e (b) a prevenção de novas situações negativas e a fuga de situações negativas que já existam. A partir de tal raciocínio, a OMR tem como objetivo investigar como o comportamento humano pode assumir essas duas formas frente às diferentes situações.

Dweck e Elliot (1983) iniciaram a discussão sobre a OMR ao criticar a utilização da inteligência como único fator que influenciaria o desempenho em situações de alcance de metas. $\mathrm{O}$ interesse era a motivação para metas de realização, uma vez que acreditavam que esse tipo de motivação influenciaria o desempenho e o sucesso de crianças em sala de aula. Foi proposto o estudo dos fatores que afetam a adoção e a busca por metas, analisando a probabilidade de uma meta ambiciosa ser perseguida, o quão vigorosamente é perseguida e por quanto tempo é perseguida.

Apesar das críticas às teorias de inteligência, Dweck (1986) posteriormente fez uso delas para explicar como os diferentes tipos de inteligência de um indivíduo influenciariam a sua orientação para metas. A partir de então, o termo "orientação para metas" passou a ser utilizado para se referir a metas de realização. $\mathrm{O}$ autor propôs a distinção entre metas de aprendizado, que se referem ao desejo do indivíduo de aprimorar suas competências e/ ou aprender coisas novas, e metas de desempenho, que tem como objetivo demonstrar competência perante os outros e evitar julgamentos negativos.

Simultaneamente, Nicholls (1984) estava investigando o conceito de orientação para metas no ambiente educacional. O interesse era o comportamento de realização, que ele define como o comportamento em que o objetivo é desenvolver ou demonstrar - para si e para os outros - grande habilidade, ou apenas evitar demonstrar pouca habilidade. Foi proposto que tais diferenças individuais poderiam ser compreendidas por meio de duas categorias: orientados para tarefa e orientados para o ego. Os primeiros julgam suas habilidades considerando a própria habilidade no passado e o quanto avançaram no domínio da tarefa, enquanto os últimos consideram o seu esforço/desempenho em relação aos outros.

Dweck (1986) e Nicholls (1984) concordam com o fato de que indivíduos adotam metas particulares (às quais eles se referem como metas de realização ou orientação para metas) em uma situação de demanda. Tais premissas serviram como base para o aprimoramento da teoria da OMR, que foi ganhando, ao longo dos anos, cada vez mais espaço entre as teorias de metas e de motivação. Atualmente ainda não há consenso com relação à definição da OMR, porém Elliot e Thrash (2001) afirmam que, geralmente, os teóricos utilizam duas abordagens básicas: 1. Descrição em termos do propósito pelo qual alguém se engaja no comportamento de realização; e 2. Caracterização de um conjunto integrado de variáveis que juntas criam uma orientação para a realização de metas de desempenho. De forma geral, Schunk (2001) define a OMR como um padrão integrado de crenças que levam as pessoas a encarar, engajar-se e responder a tarefas e situações de maneiras específicas. Quando querem alcançar uma meta, as pessoas apresentam diferentes orientações ou modos de agir em direção à conquista delas, de forma que tal orientação guia os comportamentos, cognições e afetos do indivíduo (Elliot, 2005).

Segundo Pintrich, Conley e Kempler (2003), a OMR inclui a finalidade da realização da meta (maestria ou desempenho) e os padrões e critérios que os indivíduos podem usar para definir suas metas (padrões autorreferentes ou comparativos). Metas de maestria refletem a crença no esforço que leva ao sucesso, focando no desenvolvimento de competências, aprendizagem, compreensão da tarefa/ trabalho e padrões autorreferentes de aprimoramento. Pessoas com a orientação de maestria seriam mais focadas na priorização de metas amplas (ao invés de restritas) ao realizar tarefas. Por outro lado, a orientação de desempenho foca na habilidade individual do sujeito e no senso 
de autovalor. Pessoas com uma orientação de desempenho mais elevada se preocupam em se sair melhor que os outros nas tarefas, superar as normas e receber reconhecimento pessoal (Lee, McInerney, Liem, \& Ortiga, 2010). De forma geral, metas de maestria focam no desenvolvimento de competências e na maestria das tarefas, com orientação intrapessoal, enquanto metas de desempenho são associadas à demonstração de competência em relação aos outros, com orientação interpessoal (Elliot, 1999).

Contudo, a compreensão da forma pela qual as orientações de maestria e desempenho se apresentam nos indivíduos representa uma questão de intenso debate. A compreensão do comportamento de alcance e suas consequências exige atenção não só às metas dos indivíduos, mas também às necessidades/motivos subjacentes que tais metas atendem (Thrash \& Hurst, 2008). Nesse contexto, a energização e a direção do comportamento do indivíduo podem tender para a aquisição de competência ou evitação de incompetência (Elliot \& Dweck, 2005), representados pelas dimensões de aproximação (approach) e evitação (avoidance) da OMR.

Nesse sentido, as orientações de maestria e desempenho se expressam a depender da necessidade/motivo de aproximação ou evitação do indivíduo. $\mathrm{O}$ modelo pioneiro que considerou tais dimensões foi o de Elliot e Harackiewicz (1996), conhecido como trifatorial. Nesse modelo, foi expandida a dicotomia convencional maestria-desempenho a partir da consideração de três componentes de aproximação e evitação na orientação de desempenho: 1. maestria; 2 . aproximação de desempenho; 3. evitação de desempenho.

Várias pesquisas encontraram evidências para o modelo dos três fatores (e.g. Elliot \& Church, 1997; Elliot, McGregor, \& Gable, 1999; VandeWalle, 1997; VandeWalle, Ganesan, Challagalla, \& Brown, 2000). Posteriormente Elliot (1999) propôs que a orientação de maestria também deveria ser dividida em dimensões de aproximação e evitação, argumentando que seria necessário abarcar o amplo espectro de esforços baseados em competências. Sendo assim, Elliot e McGregor (2001) propuseram que, para cada orientação (maestria e desempenho), dimensões de aproximação e evitação sejam consideradas, o que é conhecido como o modelo 2x2, conforme disposto na Figura 1. Na orientação de aproximação de maestria, as pessoas focam no desenvolvimento de competências, aprendizado, compreensão da tarefa/trabalho e priorizam padrões autorreferentes de aprimoramento. Por outro lado, na orientação de evitação de maestria as pessoas evitam demonstrar que não compreendem algo, que têm dificuldade de aprender ou que não conseguem dominar uma tarefa. Já as metas de desempenho são relacionadas ao desejo de um indivíduo de se sair melhor que os outros e de ser reconhecido por suas competências. A dimensão de evitação se associa ao medo de parecer incompetente no trabalho (Lee et al., 2010).

\begin{tabular}{|c|c|c|c|}
\hline & & \multicolumn{2}{|c|}{ Definição } \\
\hline & & $\begin{array}{c}\text { Absoluta/intrapessoal } \\
\text { (maestria) }\end{array}$ & $\begin{array}{c}\text { Normativa/interpessoal } \\
\text { (desempenho) }\end{array}$ \\
\hline \multirow{2}{*}{ Valência } & $\begin{array}{c}\text { Positiva } \\
\text { (procura sucesso) }\end{array}$ & $\begin{array}{c}\text { Aproximação } \\
\text { Maestria }\end{array}$ & $\begin{array}{l}\text { Aproximação } \\
\text { Desempenho }\end{array}$ \\
\hline & $\begin{array}{c}\text { Negativa } \\
\text { (evita fracasso) }\end{array}$ & $\begin{array}{l}\text { Evitação } \\
\text { Maestria }\end{array}$ & $\begin{array}{c}\text { Evitação } \\
\text { Desempenho }\end{array}$ \\
\hline
\end{tabular}

Fonte. Elliot e McGregor, 2001

Figura 1. Modelo 2x2 de Orientação para Alcance de Metas

A partir do modelo 2x2, Elliot e McGregor (2001) propuseram o Achievement Goal Questionnaire (AGQ), ou Questionário de Metas de Realização. Além de demonstrar empiricamente a existência de distinção entre os quatro fatores, os autores também demonstraram como cada um dos quatro tipos de orientação se relacionava a diferentes antecedentes e consequentes. Estudos posteriores (Conroy, Elliot, \& Hofer, 2003; Elliot \& Murayama, 2008; Finney, Pieper, \& Barron, 2004; Pastor, Barron, Miller, \& Davis, 2007) ajudaram a reforçar a validade teórica e empírica do modelo por meio de análises fatoriais confirmatórias, de validade discriminante, confiabilidade, modelagem por equações estruturais e análise de clusters. Os resultados de tais estudos contribuíram para atestar a prevalência do modelo de quatro fatores sobre o seu antecessor, o modelo trifatorial, fazendo com que o 2x2 seja o mais sofisticado e mais utilizado na área atualmente (Van Yperen \& Renkema, 2008).

Embora a OMR apresente uma tradição de pesquisa que data desde a década de 80 , apenas na segunda metade da década de 90 e principalmente a partir dos anos 2000 a teoria começou a ser investigada com mais frequência 
no ambiente laboral. Inicialmente, os estudos enfatizaram a relação entre OMR e o desempenho em vendas (e.g. Kohli, Shervani, \& Challaglla, 1998; Sujan, Weitz, \& Kumar, 1994; VandeWalle, Brown, Cron, \& Slocum, 1999). Mais recentemente, pesquisas investigaram a relação entre OMR e outras áreas do comportamento organizacional, tais como treinamento (ex. Kozlowski et al., 2001; Rogers \& Spitzmüeller, 2009), liderança (Yee, Lee, Yeung, \& Cheng, 2013), motivação (Dysvik \& Kuvaas, 2013), envelhecimento da força de trabalho (Heidemeier \& Staudinger, 2015), orientação para metas de equipes de trabalho (Gong, Kim, Lee, \& Zhu, 2013; Van Mierlo \& Van Hooft, 2015), satisfação na carreira e comprometimento organizacional (Joo \& Park, 2009), adaptabilidade de carreira (Tolentino et al., 2014), resultados na carreira (Godshalk \& Sosik, 2003) e treinamento, desempenho e busca por feedback (DeShon \& Gillespie, 2005).

Com relação ao contexto brasileiro, é desejável que a inexistência de estudos que relacionam a OMR com aspectos do contexto laboral/organizacional seja revertida, principalmente devido à capacidade da OMR de responder questões frequentes nas organizações. Uma delas consiste nas possíveis implicações da OMR no estabelecimento e cumprimento de metas no trabalho (Farr, Hofmann, \& Fingenbach, 1993). Outra questão concerne o porquê de alguns indivíduos procurarem se aprimorar continuamente, enquanto outros se dão por satisfeitos em seguir a vida sempre com o mesmo conjunto de conhecimentos e habilidades (DeShon \& Gillespie, 2005). Uma terceira questão diz respeito aos motivos pelos quais alguns indivíduos apreciam e buscam desafios, enquanto outros evitam desafios ou apresentam comportamentos de autossabotagem para evitá-los. Diante de tais questões, enfatiza-se a importância do estudo da OMR no Brasil e, por conseguinte, a necessidade de adaptação do instrumento de medida da OMR para o contexto laboral brasileiro.

Uma das primeiras tentativas de mensurar a OMR no contexto do trabalho foi feita por VandeWalle (1997), que desenvolveu um instrumento trifatorial por meio da integração das propostas de Dweck (1986) e Elliot e Harackiewicz (1996). Estudos posteriores com o referido instrumento (Brett \& VandeWalle, 1999; VandeWalle, 1997) demonstraram que as metas de maestria se relacionavam com resultados adaptativos no trabalho, enquanto as metas de aproximação de desempenho eram menos deletérias que as de evitaçãodo desempenho.

Baranik et al. (2007) propuseram a atualização do modelo trifatorial de VandeWalle por meio da construção do modelo 2x2 de Metas de Realização no Trabalho. O instrumento desses autores aborda o modelo de quatro fatores de Elliot e McGregor (2001) ao incluir a dimensão de evitação da maestria no contexto de trabalho, uma vez que a avaliação de tal aspecto pode ser importante devido à sua potencial presença nas organizações. Os autores indicam que a evitação de maestria costuma ser uma orientação comum entre empregados recém-contratados que apenas focam em aprender os aspectos básicos da sua função, ou de empregados mais antigos, que focam em não perder o conhecimento e as habilidades relacionadas ao seu trabalho.

Para a construção da escala $2 \times 2$, Baranik et al. (2007) utilizaram 12 itens do instrumento anterior de VandeWalle (1997) para representar as dimensões de aproximação de maestria (MAP), aproximação de desempenho (PAP) e evitação de desempenho (PAV). Para a dimensão de evitação de maestria (MAV), foram escritos novos itens, a partir da definição operacional do conceito proposta por Elliot e McGregor (2001). No total, a versão inicial da escala apresentava 23 itens, com respostas indo de 1 (é totalmente falso ao meu respeito) a 7 (é totalmente verdadeiro a meu respeito).

O modelo $2 \times 2$ tem apresentado evidências de validade em pesquisas anteriores, como a de Finney et al. (2004), que realizou um estudo com 2.111 participantes e conduziram uma análise fatorial confirmatória do Achievement Goal Questionnaire (AGQ), encontrando evidências de um melhor ajuste do modelo ao se considerar os quatro fatores, ao invés de apenas três. Elliot e Murayama (2008), por sua vez, analisaram a validade do modelo $2 \times 2$ e também mostraram que seu ajuste é melhor em comparação ao modelo trifatorial. O estudo de Conroy et al. (2003) sobre o AGQ, no contexto esportivo, demonstrou que, em comparação com outros modelos, o 2x2 apresenta o melhor ajuste. Por fim, mais recentemente duas revisões relevantes acerca do papel da orientação para metas de realização no contexto de trabalho e que encontram evidências favoráveis para a escala de Baranik et al. (2007) foram realizadas. A primeira visa discutir a conceptualização do modelo $2 \times 2$ por meio de dados obtidos sobre a orientação dominante de trabalhadores (Van Yperen \& Orehek, 2013). A segunda verifica as diferenças entre trabalhadores jovens $\mathrm{e}$ mais velhos, além de construir uma rede nomológica de evidências para a evitação de maestria, a nova dimensão abordada pela escala (Baranik, Lau, Stanley, Barron, \& Lance, 2013). Diante de tais evidências, a escala $2 \times 2$ de Baranik et al. (2007) foi concebida como o instrumento mais compreensivo da OMR adaptado ao contexto laboral, sendo, portanto, escolhida pelo presente estudo para ser traduzida, adaptada e validada para o contexto brasileiro.

\section{Método}

\section{Participantes}

Participaram do estudo 358 trabalhadores da região Centro-Oeste do Brasil, situados em diversas áreas de atividade e setores da economia, sendo 33\% homens e $67 \%$ mulheres, com idades entre 17 e 64 anos $(M=29$ anos; $D P=9,48)$. Dentre as atividades profissionais dos participantes, as de maior frequência foram técnicos/ 
analistas administrativos, estagiários, vendedores e funcionários públicos. Porém, houve também microempreendedores, artesãos, recepcionistas, atendentes, operadores de telemarketing, entre outros. Portanto, a maior parte dos participantes estava envolvida com atividades relacionadas ao terceiro setor. A maioria estava cursando ensino superior (87,3\%), incluindo-se aqueles que cursavam uma segunda graduação. No tocante ao estado civil, $57 \%$ da amostra era solteira e $39,1 \%$ era casada ou estava em união estável. O tempo de atuação dos participantes no mercado do trabalho variou entre menos de $1 \mathrm{e}$ 43 anos $(M=8,4 ; D P=8,2)$, sendo a mediana igual a 5 anos. Dos que estavam trabalhando numa organização, o tempo de trabalho variou entre menos de 1 e 38 anos $(M=3,8 ; D P=5,5)$.

\section{Instrumentos}

Foi utilizado o questionário de Metas de Realização no Trabalho modelo $2 \times 2$ de Baranik et al. (2007). A escala original em inglês apresenta 23 itens e quatro fatores (aproximação/busca de maestria - MAP, evitação/fuga de maestria - MAV, aproximação/busca de desempenho PAP, evitação/fuga de desempenho - PAV), cujos índices de confiabilidade variam de 0.69 a 0.82 . O fator da evitação/fuga de maestria apresenta 11 itens, enquanto os demais apresentam quatro itens cada. As escalas de resposta vão de 1 (não se parece em nada comigo) a 7 (se parece muito comigo).

\section{Procedimentos}

O instrumento original foi traduzido para o português por um psicólogo bilíngue. A tradução obtida foi revertida para o inglês por um tradutor/revisor profissional, de língua nativa inglesa e com fluência na língua portuguesa (back-translation). Após esse procedimento, foi verificada a equivalência semântica e sintática das versões. Foram feitos pequenos ajustes com relação a algumas palavras, de forma a equiparar as duas versões. A validação semântica foi realizada com sete estudantes de Psicologia, de períodos distintos. O instrumento mostrou-se de fácil compreensão e não foram necessários ajustes adicionais. A coleta de dados ocorreu de forma coletiva, no formato físico (em papel). Os participantes foram recrutados em salas de aula de cursos de graduação e pós-graduação. Foram convidados para participar os alunos que exerciam alguma atividade laboral, incluindo estágio. Àqueles que aceitaram participar, foi informado o objetivo geral do estudo, assim como os direitos envolvidos na participação voluntária. Todos os procedimentos éticos necessários foram atendidos. A coleta foi realizada no local de estudo dos participantes, após o final da aula, com prévio consentimento das autoridades responsáveis.

\section{Análise de Dados}

A escala traduzida e adaptada foi submetida à análise fatorial confirmatória (AFC). Para tanto, foi utilizado o software AMOS, extensão do SPSS para a Modelagem por Equações Estruturais (MEE). Os casos com dados omissos eram quase todos assistemáticos e totalizaram menos de $5 \%$, portanto foram substituídos pela média. Apenas um caso da amostra inicial foi excluído por ter apresentado mais de $5 \%$ de missings. Os casos extremos univariados que apresentaram problemas em dois itens ou mais foram excluídos, totalizando quatro casos. Os casos extremos multivariados foram identificados com base na distância de Mahalanobis, o que resultou na exclusão de sete casos. No total, 12 casos foram excluídos, de forma que a amostra original continha 370 casos e, depois das exclusões, sobraram 358 casos, como indicado na seção sobre a amostra. Os indicadores apontaram que a maioria das variáveis tinha distribuição anormal. De forma a tentar atender esse pressuposto, foram testados dois tipos de transformação: logaritmo na base 10 e 1/X, de acordo com as recomendações de Tabachinick e Fidel (2001) para casos de skewness positiva. Porém, observou-se que as transformações não geraram melhoras significativas. Sendo assim, optou-se por utilizar o bootstrap. Contudo, cabe ainda ressaltar que tanto a análise fatorial exploratória como confirmatória são relativamente robustas contra violações de normalidade (Gorsuch, 1983, citado em Laros, 2004), principalmente se não houver violação concomitante a outros pressupostos, como é o presente caso. O método de estimação utilizado foi o da máxima verossimilhança (Maximum likelihood) e para o teste de adequação do modelo estrutural foram utilizados o SRMR (valor abaixo de 0,06 indica ajuste) GFI e o CFI (valores superiores a 0,90 indicam ajuste) e o RMSEA (com valor inferior a 0,08 para ajuste). A precisão dos fatores resultantes foi avaliada por meio do alfa de Cronbach.

\section{Resultados}

A análise fatorial confirmatória foi conduzida inicialmente com os 23 itens se agrupando em quatro fatores (MAP, MAV, PAP, PAV), o que não gerou indicadores satisfatórios de ajuste do modelo, assim como relatado por Baranik et al. (2007). Também foram testadas estruturas alternativas, como o modelo trifatorial. Porém, o ajuste do modelo trifatorial não demonstrou ser melhor do que o de quatro fatores. Dessa forma, considerando indicações dos Modification Indices, da análise das cargas fatoriais mais baixas (itens com cargas fatoriais abaixo de 0,40 foram excluídas) e dos resultados obtidos na pesquisa dos autores foram excluídos cinco itens do fator evitação/fuga de maestria: MAV1, MAV3, MAV7, MAV8 e MAV10. Esses mesmos itens apresentaram problemas de ajuste na pesquisa original e também foram excluídos. Assim como na presente pesquisa, os Modification Indices sugeriram relações de alguns desses itens com outros fatores que não o seu fator de origem, o que pode indicar que tais itens representam mais de um conceito. $\mathrm{O}$ 
grande número de possíveis covariâncias entre os erros desses seis itens também sugere que eles podem estar representando um construto diferente daquele apresentado pela evitação/fuga de maestria.

Segundo Baranik et al., (2007), o conteúdo dos itens MAV7 e MAV10 pode sugerir que eles compartilhem variância relacionada a autoeficácia (MAV7: "Eu evito assumir novas tarefas no trabalho quando eu não tenho certeza de que vou ser capaz de aprender ou dominar tais tarefas"), enquanto os itens usados para acessar a evitação/fuga de maestria em geral não o fazem (MAV11: "No trabalho, eu tento evitar que o meu desempenho nas tarefas requeridas para a minha função seja ruim"). Já os itens MAV3 e MAV9, por sua vez, apresentam redação redundante e quase idêntica (MAV3: "Eu espero ser capaz de dominar habilidades suficientes para ser competente na minha função"; MAV9: "Eu espero ser capaz de manter habilidades suficientes para ser competente na minha função"), de forma que a covariância de seus erros gerou um aumento substancial no ajuste do modelo. Sendo assim, apenas a presença de um dos dois itens (MAV9) seria necessária. Por fim, os autores apontam que o item MAV1 pareceu compartilhar variância com itens que foram escritos para acessar a evitação/fuga de desempenho (MAV1: "Minha principal meta no trabalho é evitar falhar nas tarefas requeridas para a minha função").

$O$ resultado encontrado no presente estudo replica os resultados do estudo original, o que reforça as evidências para a estrutura fatorial de 18 itens proposta pelos autores. A Figura 2 representa a solução fatorial proposta, com os fatores latentes e seus respectivos indicadores. Como consta na Figura 2, foram estabelecidas quatro covariâncias entre erros, sendo três no fator de aproximação de desempenho e um no fator de evitação de maestria.

$\mathrm{Na}$ Figura 2, também estão dispostas as cargas fatoriais estimadas, que variaram de 0,26 a 0,81 . As replicações bootstrap indicaram estabilidade de estimação das cargas fatoriais, o que contribui para a adequação de ajuste do modelo apesar da ausência de normalidade. Desde as primeiras análises (com todos os itens), os dois fatores de desempenho e o fator de aproximação de maestria já se mostravam estáveis e com cargas com valores significativos nos fatores latentes. Depois da retirada dos cinco itens problemáticos, o fator evitação de maestria obteve resultados semelhantes.

Os índices de ajuste do modelo com quatro fatores e 18 itens foi: $\mathrm{CMIN} / \mathrm{DF}=2,12$; $\mathrm{SRMR}=0,06$; $\mathrm{GFI}=0,92$; $\mathrm{CFI}=0,90$; RMSEA=0,05. Conforme critérios utilizados na literatura (Pilati \& Laros, 2007), os índices de ajuste do modelo são satisfatórios e indicam um alto grau de ajuste do modelo aos dados. Tais resultados sugerem que a análise fatorial confirmatória foi robusta à assimetria dos dados e que os itens são bons indicadores dos construtos latentes dessa medida.
Apesar do bom ajuste do modelo, os índices de confiabilidade dos fatores se apresentaram como razoáveis, variando entre 0,60 e 0,77 , conforme Tabela 1 . Isso pode ser explicado pela tendência do alfa de Cronbach a cair quando os itens não apresentam coeficientes de caminho (path) iguais (Reuterberg \& Gustafsson, 1992, citado em Baranik et al., 2007). A escala original também não apresentou índices de confiabilidade excelentes (entre 0,69 e 0,82 ), o que pode explicar parcialmente os resultados obtidos no presente estudo. Sendo assim, no caso da escala em questão, enfatiza-se a importância dos índices de ajuste do modelo obtidos na análise fatorial confirmatória, citados acima.

Os resultados encontrados no presente estudo são compatíveis com estudos anteriores acerca da validade do modelo 2x2 (Conroy et al., 2003; Elliot \& Murayama, 2008; Finney et al., 2004), ao demonstrar um melhor ajuste do modelo de quatro fatores, em comparação com o modelo trifatorial. Diante disso, os resultados encontrados neste estudo se alinham com o direcionamento atual das pesquisas em OMR, reforçando a importância do instrumento para o contexto de trabalho brasileiro. A escala final em português encontra-se no Anexo A.

\section{Discussão}

O objetivo deste estudo foi traduzir, adaptar e identificar evidências de validade para o instrumento proposto por Baranik et al. (2007), o modelo 2x2 de Metas de Realização no Trabalho. Diante dos resultados descritos anteriormente, conclui-se que o objetivo foi alcançado, disponibilizando-se assim o primeiro instrumento para mensuração de metas de realização no trabalho para o contexto brasileiro.

O instrumento brasileiro apresenta os mesmos itens da versão reduzida de 18 itens de Baranik et al. (2007) e os resultados obtidos no presente estudo se assemelham aos obtidos pelos autores. A utilização do instrumento proposto facilitará o desenvolvimento da pesquisa no Brasil sobre alcance de metas no trabalho e apresenta implicações teóricas e práticas importantes. Algumas das implicações teóricas envolvem a disseminação de uma teoria que gera muitos frutos na literatura internacional, mas que ainda é incipiente no Brasil. Pesquisadores estrangeiros têm encontrado resultados relevantes no que diz respeito à influência da OMR nas ações humanas e, principalmente, no desempenho humano. Uma vez que, no Brasil, tem-se disponível uma escala para mensuração da OMR, é possibilitado o diálogo com pesquisas internacionais para se compreender melhor o comportamento e a motivação das pessoas no trabalho e nas organizações. Adicionalmente, abre-se um novo mundo de possibilidades no que diz respeito a relações de antecedência e consequência entre a OMR e outras variáveis, individuais e contextuais, do ambiente laboral. 


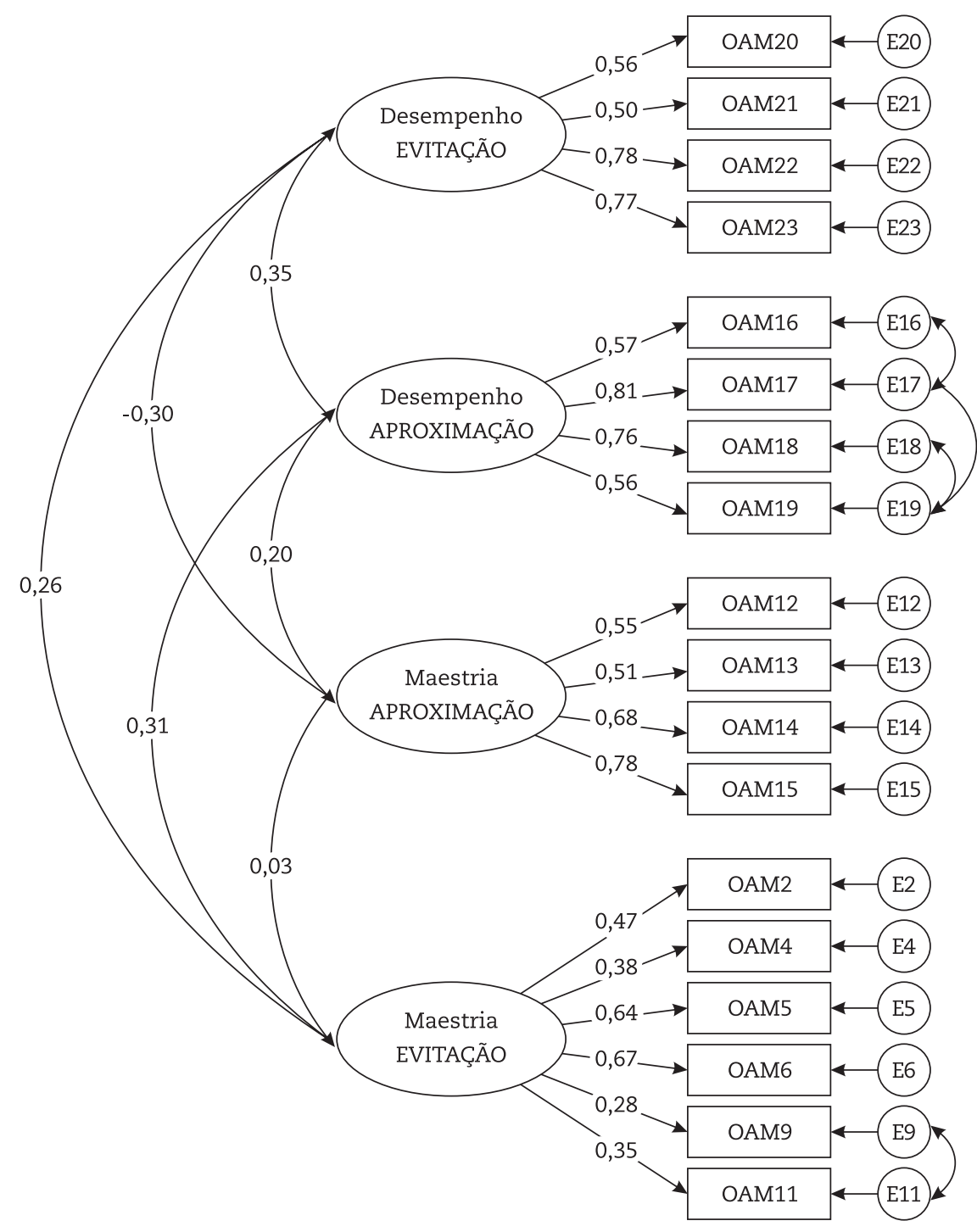

Figura 2. Modelo estrutural final da AFC para a Escala de Metas de Realização no Trabalho

Tabela 1

Alfas de Cronbach - Escala Original e Escala Adaptada

\begin{tabular}{lcccc}
\hline & MAP & MAV & PAP & PAV \\
\hline Escala adaptada (Português) & 0.71 & 0.60 & 0.77 & 0.74 \\
Escala original (Inglês) & 0.82 & 0.82 & 0.69 & 0.78 \\
\hline
\end{tabular}

Uma implicação prática da proposição do presente instrumento é a oportunidade de investigar aspectos fundamentais do que motiva as pessoas a perseguirem metas no trabalho, permitindo analisar as diferentes formas pelas quais um trabalhador busca o alcance de uma meta e qual orientação perpassa suas ações no trabalho. A partir de tal diagnóstico, seria possível realocar as pessoas em funções mais adequadas, designar tarefas mais compatíveis com seus perfis e, até mesmo, orientar e treinar as lideranças para trabalhar com os diferentes tipos de orientação que as pessoas podem vir a apresentar.
Uma das limitações a ser apontada neste estudo é a sua restrição à validação de estrutura da escala, sendo desejável uma ampliação em direção a validações preditiva e discriminante e à construção de uma rede nomológica. Além disso, destaca-se a necessidade de outros estudos que contribuam para a confirmação da estrutura fatorial da escala no contexto brasileiro, assim como o mapeamento da dinâmica de fatores que levam o trabalhador brasileiro a apresentar determinada orientação para metas de realização em contextos específicos. 


\section{Referências}

Baranik, L. E., Barron, K. E., \& Finney, S. J. (2007). Measuring goal orientation in a work domain: Construct validity evidence for the 2X2 framework. Educational and Psychological Measurement, 67(4) 697-718. doi: 10.1177/0013164406292090

Baranik, L. E., Lau, A. R., Stanley, L. J., Barron, K. E., \& Lance, C. E. (2013). Achievement goals in organizations: Is there support for mastery-avoidance? Journal of Managerial Issues, 25(1), 46-61.

Brett, J. F., \& VandeWalle, D. (1999). Goal orientation and goal content as predictors of performance in a training program. Journal of Applied Psychology, 84(6), 863-873. doi: 10.1037/0021-9010.84.6.863

Conroy, D. E., Elliot, A. J., \& Hofer, S. M. (2003). A 2x2 achievement goals questionnaire for sport: Evidence for factorial invariance, temporal stability, and external validity. Journal of Sport and Exercise Psychology, 25(4), 456-476.

DeShon, R. P., \& Gillespie, J. Z. (2005). A motivated action theory account of goal orientation. Journal of Applied Psychology, 90(6)90, 1096-1127. doi: 10.1037/0021-9010.90.6.1096

Dweck, C. S. (1986). Motivational processes affecting learning. American Psychologist, 41(10), 1040-1048. doi: 10.1037/0003066X.41.10.1040

Dysvik, A., \& Kuvaas, B. (2013), Intrinsic and extrinsic motivation as predictors of work effort: The moderating role of achievement goals. British Journal of Social Psychology, 52(3), 412-430. doi: 10.1111/j.2044-8309.2011.02090

Dweck, C. S., \& Elliott, E. S. (1983). Achievement motivation. Em P. Mussen \& E. M. Hetherington (Eds.), Handbook of child psychology (pp. 643-691). New York: Wiley.

Elliot, A. J. (1999). Approach and avoidance motivation and achievement goals. Educational Psychologist, 34(3), 169-189. doi:10.1207/ s15326985ep3403_3

Elliot, A. J. (2005). A conceptual history of the achievement goal construct. Em A. Elliot \& C. Dweck (Eds.), Handbook of competence and motivation (pp. 52-72). New York: Guilford Press.

Elliot, A. J. (2006). The hierarchical model of approach-avoidance motivation. Motivation and Emotion, 30(2), 111-116. doi: 10.1007/s11031006-9028-7

Elliot, A. J., \& Church, M. (1997). A hierarchical model of approach and avoidance achievement motivation. Journal of Personality and Social Psychology, 72(1), 218-232. doi: 10.1037/0022-3514.72.1.218

Elliot, A. J., \& Dweck, C. S. (Eds.) (2005). Handbook of competence and motivation. New York: Guilford Press.

Elliot, A. J., \& Harackiewicz, J. M. (1996). Approach and avoidance goals and intrinsic motivation: A mediation analysis. Journal of Personality and Social Psychology, 70(3), 525-538. doi: 10.1037/0022-3514.70.3.461

Elliot, A. J., \& McGregor, H. A. (2001). A 2x2 achievement goal framework. Journal of Personality and Social Psychology, 80(3), 501-519. doi: 10.1O37//OO22-3514.80.3.501.

Elliot, A. J., \& Thrash, T. M. (2001). Achievement goals and the hierarchical model of achievement motivation. Educational Psychology Review, 13(2), 139-156. doi: 10.1023/A:1009057102306

Elliot, A. J., McGregor, H. A., \& Gable, S. (1999). Achievement goals, stufy strategies, and exam performance: A mediational analysis. Journal of Educational Psychology, 91(3), 549-563. doi: 10.1037/0022-0663.91.3.549

Elliot, A. J., \& Murayama, K. (2008). On the measurement of achievement goals: Critique, illustration, and application. Journal of Educational Psychology, 100(3), 613-628. doi: 10.1037/0022-0663.100.3.613

Farr, J. L., Hofmann, D. A., \& Fingenbach, K. L. (1993). Goal orientation and action control theory: Implications for industrial and organizational psychology. International Review of Industrial and Organizational Psychology, 8(2), 193-232.

Finney, S. J., Pieper, S. L., \& Barron, K. E. (2004). Examining the psychometric properties of the achievement goal questionnaire in a general academic context. Educational and Psychological Measurement, 64(2), 365-382. doi: 10.1177/0013164403258465

Godshalk, V. M., \& Sosik, J. J. (2003). Aiming for success: The role of learning goal orientation in mentoring relationships. Journal of Vocational Behavior, 63(3), 417-437. doi: 10.1016/S0001-8791(02)00038-6

Gong, Y. P., Kim, T. Y., Lee, D. R., \& Zhu, J. (2013). A multilevel model of team goal orientation, information exchange and creativity. Academy of Management Journal, 56(3), 827-851. doi: 10.5465/amj.2011.0177

Heidemeier, H., \& Staudinger, U. M. (2015). Age differences in achievement goals and motivational characteristics of work in an ageing workforce. Ageing and Society, 35(4), 809-836. doi:10.1017/S0144686X13001098

Joo, B., \& Park, S. (2009). The impacts of goal orientation, organizational learning culture and developmental feedback on career satisfaction, organizational commitment and turnover intention. Em T. J. Chermack, J. Storberg-Walker \& C. M. Graham (Eds.), Academy of Human Resource Development 2009 Conference Proceedings (pp. 1353-1382). Washington, DC: AHRD.

Kozlowski, S. W. J., Toney, R. J., Mullins, M. E., Weissbein, D. A., Brown, K. G., \& Bell, B. S. (2001). Developing adaptability: A theory for the design of integrated-embedded training systems. Em E. Salas (Ed.), Advances in human performance and cognitive engineering research (pp. 59-123). Amsterdam: Elsevier Science/JAI Press.

Kohli, A. K., Shervani, T. A., \& Challaglla, G. N. (1998). Learning and performance orientation of salespeople: The role of supervisors. Journal of Marketing Research, 35(2), 263-274. doi: 10.2307/3151853

Laros, J. A. (2004). O uso da análise fatorial: algumas diretrizes para pesquisadores. Em L. Pasquali (Ed.), Análise fatorial para pesquisadores (pp. 147-170). Petrópolis: Vozes.

Lee, J. Q., McInerney, D. M., Liem, A. D., \& Ortiga Y. P. (2010). The relationship between future goals and achievement goal orientations: An intrinsic-extrinsic motivation perspective. Contemporary Educational Psychology, 35(4), 264-279. doi: 10.1016/j.cedpsych.2010.04.004

Nicholls, J. (1984). Conceptions of ability and achievement motivation. Em R. Ames \& C. Ames (Eds.), Research on motivation in education: Student motivation (Vol. 1, pp. 39-73). New York: Academic Press.

Pastor, D. A., Barron, K. E., Miller, B. J., \& Davis, S. L. (2007). A latent profile analysis of college student's achievement goal orientation. Contemporary Educational Psychology, 32(1), 8-47. doi: 10.1016/j.cedpsych.2006.10.003

Pilati, R., \& Laros, J. A. (2007). Modelos de equações estruturais em psicologia: conceitos e aplicações. Psicologia: Teoria e Prática, 23(2), 205216. doi: 10.1590/S0102-37722007000200011

Pintrich, P. R., Conley, A. M., \& Kempler, T. M. (2003). Current issues in achievement goal theory and research. International Journal of Educational Research, 39(4-5), 319-337. doi: 10.1016/j.ijer.2004.06.002 
Rogers, A. M., \& Spitzmüller, C. (2009). Individualism/collectivism and the role of goal orientation in organizational training. International Journal of Training and Development, 13(3), 185-201. doi: 10.1111/j.1468-2419.2009.00326.x

Santos, A. A. A., Alcará, A. R., \& Monteiro, R. M. (2012). A motivação para aprender na perspectiva da teoria de metas de realização e teoria de autodeterminação. Em E. Boruchovitch, A. A. A. Santos \& E. Nascimento (Eds.). Avaliação psicológica nos contextos educativo e psicossocial (pp.149-179). São Paulo: Casa do Psicólogo.

Santos, A. A. A., Alcará, A. R., \& Zenorini, R. P. C. (2013). Estudos psicométricos da escala de motivação para a aprendizagem de universitários (EMAPRE). Fractal, 25(3), 531-546. doi: 10.1590/S1984-02922013000300008.

Santos, A. A. A., Mognon, J. F., Alcará, A. R., \& Lemos, T. H. (2011). Motivação para aprender: evidência de validade convergente entre duas medidas. Aletheia, (35-36), 36-50.

Schunk, D. H. (2001). Social cognitive theory and self-regulated learning. Em B. J. Zimmerman \& D. H. Schunk (Eds.). Self-regulated learning and academic achievement: Theoretical perspectives ( $2^{\mathrm{a}}$ Ed., pp. 125-151). Mahwah, NJ: Lawrence Erlbaum Associates, Inc.

Sujan, H., Weitz, B., \& Kumar, N. (1994). Learning orientation, working smart, and effective selling. Journal of Marketing, 58(3), 39-52. doi: $10.2307 / 1252309$

Tabachnick, B. G., \& Fidell, L. S. (2001). Using multivariate statistics. Boston: Allyn \& Bacon.

Thrash, T., \& Hurst, A. (2008). Approach and avoidance motivation in the achievement domain: Integrating the achievement motive and achievement goal traditions. Em A. J. Elliot (Ed.), Handbook of approach and avoidance motivation (pp. 217-233). New York, NY: Psychology Press.

Tolentino, L. R., Garcia, P. R. J. M., Lu, V. N., Restubog, S. L. D., Bordia, P., \& Plewa, C. (2014). Career adaptation: The relation of adaptability to goal orientation, proactive personality, and career optimism. Journal of Vocational Behavior, 84(1), 39-48. doi: 10.1016/J. Jvb.2013.11.004

Valentini, N. C., \& Rudisill, M. E. (2006). Goal orientation and mastery climate: A review of contemporary research and insights to intervention. Estudos em Psicologia (Campinas), 23(2), 159-171. doi:10.1590/S0103-166X2006000200006

VandeWalle, D. (1997). Development and validation of a work domain goal orientation instrument. Educational and Psychological Measurement, 57(6), 995-1015. doi: 10.1177/0013164497057006009

VandeWalle, D., Brown, S. P., Cron, W. L., \& Slocum, J. W. (1999). The influence of goal orientation self-regulation tactics on sales performance: A longitudinal field study. Journal of Applied Psychology, 84(2), 249-259. doi: 10.1037/0021-9010.84.2.249

VandeWalle, D., Ganesan, S., Challagalla, G. N., \& Brown, S. P. (2000). An integrated model of feedback-seeking behavior: Disposition, context, and cognition. Journal of Applied Psychology, 85(6), 996-1003. doi: 10.1037/0021-9010.85.6.996

Van Mierlo, H., \& Van Hooft, E. A. J. (2015). A group-level conceptualization of the $2 \times 2$ achievement goal framework: Antecedents and motivational outcomes. Group E Organization Management, published online before printon July 23, 2015. doi: 10.1177/1059601115592990 Recuperado de http://gom.sagepub.com/content/early/2015/07/10/1059601115592990

Van Yperen, N. W., \& Orehek, E. (2013). Achievement goals in the workplace: Conceptualization, prevalence, profiles, and outcomes. Journal of Economic Psychology, 38, 71-79. doi:10.1016/j.joep.2012.08.013

Van Yperen, N. W., \& Renkema, L. J. (2008). Performing great and the purpose of performing better than others: On the recursiveness of the achievement goal adoption process. European Journal of Social Psychology, 38(2), 260-271. doi: 10.1002/ejsp.425

Yee, R. W. Y., Lee, P. K. C., Yeung, A. C. L., \& Cheng, T. C. E. (2013). The relationships among leadership, goal orientation, and service quality in high-contact service industries: An empirical study. International Journal of Production Economics, 141(2), 452-464. doi:10.1016/j. ijpe.2011.12.012

Zenorini, R. P. C., \& Santos, A. A. A. (2010). Escala de metas de realização da motivação para aprendizagem. Interamerican Journal of Psychology, 44(2), 291-298.

Zenorini, R. P., Santos, A. A., \& Bueno, J. M. H. (2003). Escala de avaliação das metas de realização: Estudo preliminar de validação. Avaliação Psicológica, 2(2), 165-173.

Apêndice A

Escala de Metas de Realização no Trabalho

A seguir você encontrará uma série de frases que abordam aspectos da sua vida profissional. Use a chave de respostas abaixo para informar o seu grau de concordância com o significado da frase. Quanto maior o número, mais você concorda com a frase. Assinale apenas uma resposta por frase e, por favor, não deixe nenhum item sem resposta.

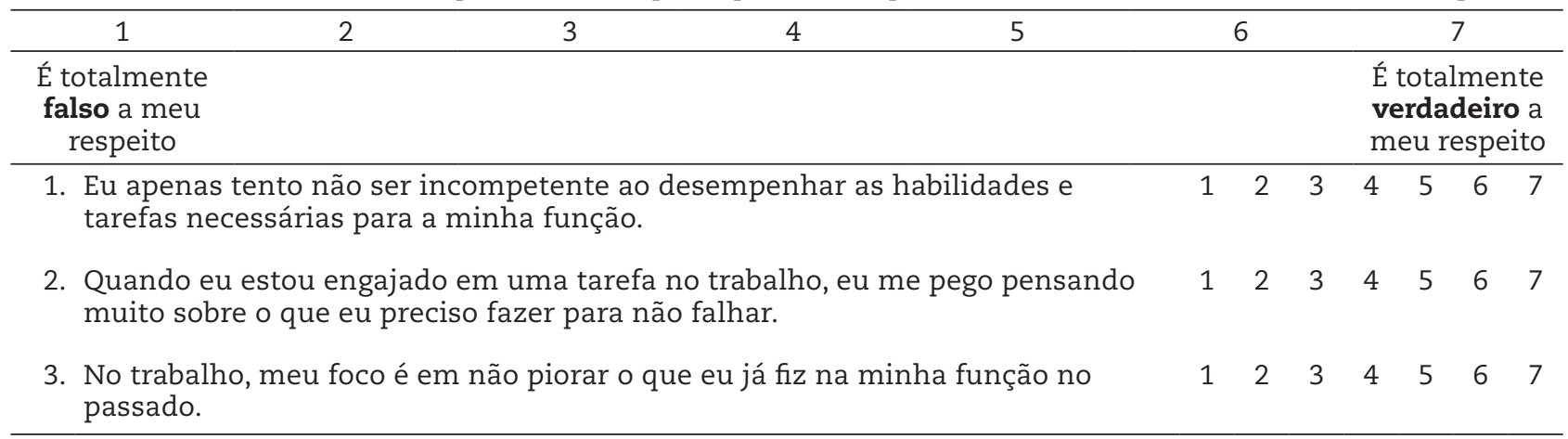


Apêndice A (continuação)

Escala de Metas de Realização no Trabalho

\begin{tabular}{|c|c|c|c|c|c|c|}
\hline 1 & 2 & 3 & 4 & 5 & 6 & 7 \\
\hline $\begin{array}{l}\text { É totalmente } \\
\text { falso a meu } \\
\text { respeito }\end{array}$ & & & & & & $\begin{array}{l}\text { É totalmente } \\
\text { verdadeiro a } \\
\text { meu respeito }\end{array}$ \\
\hline
\end{tabular}

4. Minha meta é evitar ser incompetente ao desempenhar as habilidades e

$\begin{array}{lllllll}1 & 2 & 3 & 4 & 5 & 6 & 7\end{array}$
tarefas necessárias para a minha função.

5. Eu espero ser capaz de manter habilidades suficientes para ser competente na $\quad \begin{array}{lllllllll}1 & 2 & 3 & 4 & 5 & 6 & 7\end{array}$ minha função.

6. No trabalho, eu tento evitar que o meu desempenho nas tarefas requeridas para a minha função seja ruim.

7. Eu estou disposto a escolher uma tarefa desafiante no trabalho com a qual eu $\quad \begin{array}{ccccccccc}1 & 2 & 3 & 4 & 5 & 6 & 7\end{array}$ possa aprender bastante.

8. Para mim, o desenvolvimento das minhas habilidades no trabalho é importante o suficiente para correr riscos.

9. Com frequência, eu procuro oportunidades para desenvolver novas habilidades $\quad \begin{array}{llllllll}1 & 2 & 3 & 4 & 5 & 6 & 7\end{array}$ e conhecimentos.

10. Eu gosto de tarefas difíceis e desafiadoras no trabalho, que me façam aprender $\quad \begin{array}{llllllllll}1 & 2 & 3 & 4 & 5 & 6 & 7\end{array}$ novas habilidades.

11. Eu gosto de mostrar que eu posso ter um melhor desempenho do que meus colegas de trabalho.

12. Eu prefiro trabalhar em projetos nos quais eu possa provar minhas habilidades para os outros.

13. Eu tento descobrir o que é necessário para provar minha habilidade para os outros no trabalho.

14. Eu gosto quando as pessoas no meu trabalho sabem o quanto eu estou indo bem no trabalho.

15. Eu evitaria assumir uma nova tarefa se houvesse chance de eu parecer muito incompetente para as outras pessoas.

16. Evitar mostrar pouca habilidade é mais importante para mim do que aprender uma nova habilidade.

17. Eu prefiro evitar situações no trabalho nas quais eu poderia ter um desempenho ruim.

18. Eu tenho receio de assumir uma tarefa no trabalho se o meu desempenho nela puder revelar que eu tenho poucas habilidades.

\section{Sobre as autoras}

Lígia Carolina Oliveira-Silva é Doutora em Psicologia Social, do Trabalho e das Organizações pela Universidade de Brasília e professora adjunta de Psicologia Organizacional e do Trabalho no Instituto de Psicologia da Universidade Federal de Uberlândia.

Juliana Barreiros Porto é doutora e professora adjunta do Departamento de Psicologia Social e do Trabalho e do programa de pósgraduação em Psicologia Social, do Trabalho e das Organizações da Universidade de Brasília. Lidera o Núcleo de Pesquisa em Cultura, Valores e Comportamento (CNPq) e coordena o Grupo Tamayo na UnB. 